\title{
Effect of Surface Andreev Bound States on the Bean-Livingston Barrier in d-Wave Superconductors
}

\author{
C. Iniotakis, ${ }^{1}$ T. Dahm, ${ }^{2,3}$ and N. Schopohl ${ }^{2}$ \\ ${ }^{1}$ Institute for Theoretical Physics, ETH Zurich, 8093 Zurich, Switzerland \\ ${ }^{2}$ Institut für Theoretische Physik, Universität Tübingen, \\ Auf der Morgenstelle 14, D-72076 Tübingen, Germany \\ ${ }^{3}$ Institute for Solid State Physics, University of Tokyo, Kashiwanoha, Kashiwa, Chiba 277-8581, Japan
}

(Dated: August 12, 2021)

\begin{abstract}
We study the influence of surface Andreev bound states in $d$-wave superconductors on the BeanLivingston surface barrier for entry of a vortex line into a strongly type-II superconductor. Starting from Eilenberger theory we derive a generalization of London theory to incorporate the anomalous surface currents arising from the Andreev bound states. This allows us to find an analytical expression for the modification of the Bean-Livingston barrier in terms of a single parameter describing the influence of the Andreev bound states. We find that the field of first vortex entry is significantly enhanced. Also, the depinning field for vortices near the surface is renormalized. Both effects are temperature dependent and depend on the orientation of the surface relative to the $d$-wave gap.
\end{abstract}

PACS numbers: 74.20.Rp, 74.45.+c, 74.25.Op

Formation and dynamics of vortices is of interest in such diverse fields as cosmology, liquid crystals, and superfluids [1]. It is well known that fermionic states existing inside a vortex can significantly alter the structure of a vortex line [2]. In unconventional superconductors fermionic states, so-called Andreev bound states, can also appear at the surface. In this work we show that such surface states strongly influence the entrance and pinning properties of a vortex. Entry of a vortex into a type-II superconductor is hindered by the so-called BeanLivingston (BL) surface barrier [3]. This barrier arises due to a competition of two forces acting on the vortex line: a force coming from the external magnetic field driving the vortex into the superconductor and a force of the vortex' mirror image attracting it towards the outside. As a result, the penetration field $B_{s}$ of first vortex entry is typically much larger than the lower critical field $B_{c 1}$ and becomes of the order of the thermodynamic critical field $B_{c}$ [4].

At the surface of a $d$-wave superconductor Andreev bound states exist, depending on the relative orientation of the $d$-wave gap function to the surface $[\underline{5},[6,67,8]$. It has been shown that these states split in the presence of a supercurrent running along the surface, generating a quasi-particle current directed opposite to the supercurrent 9]. At low temperatures this anomalous Meissner current even over-compensates the supercurrent, leading to a reversal of the current flow at the surface [9, 10]. Even though the Andreev bound states only exist within a coherence length $\xi$ from the surface, they are influenced by a vortex line already, when the vortex is a penetration depth $\lambda \gg \xi$ away from the surface, because the supercurrent field around the vortex is long-ranged [11]. Therefore, a modification of the BL barrier has to be expected due to the change of the free energy of the Andreev bound states as a function of the position of the vortex creating an additional force. Here, we study this modification of the BL barrier and show that it results in a sizeable increase of the penetration field and the depinning field.

We consider a superconducting half-space in the region $x \geq 0$. Both the cylindrical Fermi surface of the $d$-wave superconductor and the external magnetic field $\mathbf{B}_{0}$ shall be aligned parallel to the $z$-axis. In the following the single Abrikosov vortex is situated at the position $\mathbf{r}_{v}=\left(x_{v}, 0\right)$ on the $x$-axis, and the specular surface shall be given by the $y$-axis. Since we wish to explore Andreev bound states in an inhomogenous superconductor, Ginzburg-Landau theory is not sufficient and as a starting point the minimum theory necessary is Eilenberger theory 12, 13, 14]. In particular, we use the Riccatiparametrization [15, 16], where local equilibrium properties of the $d$-wave superconductor are determined in terms of two scalar coherence functions $a$ and $b$. To each unit vector $\hat{\mathbf{k}}$ parametrizing the Fermi surface of the superconductor corresponds a quasiparticle trajectory in real space which is parallel to the Fermi velocity $\mathbf{v}_{F}(\hat{\mathbf{k}})$. Along this trajectory, and for given Matsubara frequency $\varepsilon_{n}=(2 n+1) \pi k_{B} T$, two decoupled differential equations of the Riccati type have to be integrated

$$
\begin{aligned}
\left\langle\hbar \mathbf{v}_{F}, \nabla\right\rangle a+\left(2 \varepsilon_{n}-2 i e / c\left\langle\mathbf{v}_{F}, \mathbf{A}\right\rangle+\Delta^{*} a\right) a-\Delta & =\propto 1) \\
\left\langle\hbar \mathbf{v}_{F}, \nabla\right\rangle b-\left(2 \varepsilon_{n}-2 i e / c\left\langle\mathbf{v}_{F}, \mathbf{A}\right\rangle+\Delta b\right) b+\Delta^{*} & =0 .
\end{aligned}
$$

Here, the arguments have been omitted for brevity, and in the following we use the specific gauge, in which the gap function $\Delta$ is real and a phase gradient is absorbed into the magnetic vector potential A. Starting from known bulk values, solutions for $a(b)$ can be calculated in a stable way numerically, if the integration of the corresponding Riccati equation is performed parallel (antiparallel) to the trajectory direction. Both the gap function $\Delta(\mathbf{r}, \hat{\mathbf{k}})=\Delta_{\infty} \chi(\hat{\mathbf{k}}) \psi(\mathbf{r})$ and the magnetic vector poten- 
tial $\mathbf{A}(\mathbf{r})$ can be regarded as spatially dependent input parameters for the Riccati equations (11). In general, however, their correct selfconsistent form has to be determined from two additional conditions. One of them is given by the gap equation

$$
\Delta_{\infty} \psi(\mathbf{r})=2 \pi N_{0} V k_{B} T \sum_{\varepsilon_{n}>0}^{\omega_{c}}\left\langle\chi(\hat{\mathbf{k}}) \frac{2 a}{1+a b}\left(\mathbf{r}, \hat{\mathbf{k}}, \varepsilon_{n}\right)\right\rangle,
$$

where $N_{0}$ is the density of states in the normal phase, $V$ the interaction strength, $\omega_{c}$ the cut-off energy and $\langle\ldots\rangle$ denotes an average over the Fermi surface of the superconductor. Furthermore, the momentum dependence of the $d$-wave gap is implemented by $\chi(\hat{\mathbf{k}})=\cos 2 \phi$. The other condition regards electrodynamic selfconsistency. It can be ensured via the quasiclassical expression for the current density

$$
\mathbf{j}(\mathbf{r})=-4 \pi i e N_{0} k_{B} T \sum_{\varepsilon_{n}>0}^{\omega_{c}}\left\langle\mathbf{v}_{F}(\hat{\mathbf{k}}) \frac{1-a b}{1+a b}\left(\mathbf{r}, \hat{\mathbf{k}}, \varepsilon_{n}\right)\right\rangle
$$

together with the Maxwell equation

$$
\nabla \times \nabla \times \mathbf{A}(\mathbf{r})=\frac{4 \pi}{c} \mathbf{j}(\mathbf{r}) .
$$

The system of equations (1)-(4) is self-contained. In principle it can be used to find a fully selfconsistent solution, which is very time-consuming, however.

In the following we present a way to derive a very good approximation to the fully selfconsistent solution of Eqs. (11)-(4). We concentrate on a $d$-wave superconductor with $\kappa=\lambda / \xi \gg 1$. Furthermore the superconductor is considered to be clean, but not superclean. In other words, the effective mean free path $v_{F} \tau$ along a quasiparticle trajectory may be larger than the coherence length $\xi=\hbar v_{F} / \Delta_{\infty}$, but it should also hold $\lambda \gg v_{F} \tau$. As a starting point we then use the selfconsistent solution of Eqs. (1)-(2) in the absence of any magnetic vector potentials and currents. This solution can be found numerically comparatively easy since the problem is translationally invariant along the boundary. This solution already contains both the existence of low-energy Andreev bound states and the local suppression of the gap in the vicinity of the boundary. In a next step, we start perturbing this known selfconsistent solution by a magnetic vector potential. Due to the assumptions stated above, we can restrict ourselves to perturbations, which typically vary on the length scale $\lambda$ and are effectively homogeneous along the mean free path $v_{F} \tau$. In that case, the Riccati equations (1) can be evaluated for a constant Doppler shift in energy, $\frac{e}{c}\left\langle\mathbf{v}_{F}, \mathbf{A}\right\rangle$, along the quasiparticle trajectory. Let $\alpha$ denote the angle between the surface normal and a maximum gap direction of the $d$-wave (cf. inset of Fig. 1). For the two most symmetric cases $\alpha=0$ and $\alpha=\pi / 4$ respectively, the expansion of Eq. (3) to

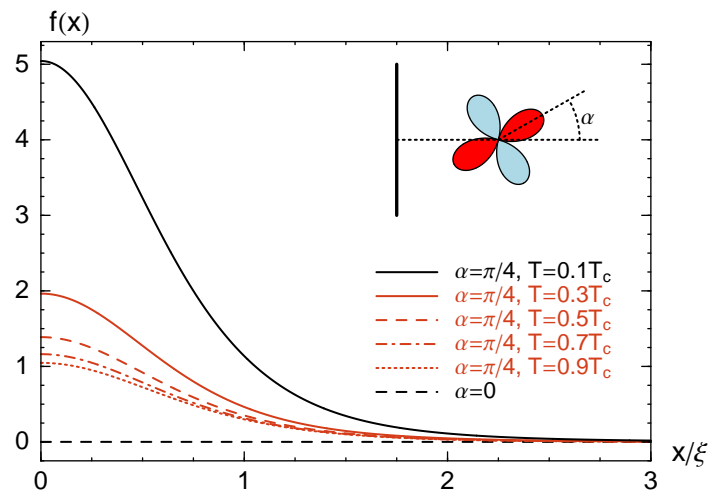

FIG. 1: (Color online) Dimensionless function $f$, determining the local current response according to Eq. (5), as a function of the distance $x$ to the boundary of a $d$-wave superconductor. Curves are shown for different temperatures and orientations. London electrodynamics, i.e. $f=0$, holds for the $d$-wave orientation $\alpha=0$ and towards the bulk. For $\alpha=\pi / 4$ the current response in the vicinity of the boundary is modified due to surface Andreev bound states. This effect is strongly enhanced for lower temperatures.

first order of the perturbing vector potential $\mathbf{A}$ yields an equation of the form

$$
\mathbf{j}(\mathbf{r})=-\frac{c}{4 \pi} \frac{1}{\lambda^{2}}[1-f(\mathbf{r})] \mathbf{A}(\mathbf{r}),
$$

where the dimensionless function $f$ on the righthand side is independent of $\lambda$ and can be determined numerically from the known fully selfconsistent solution for $\mathbf{A}=0$ mentioned above.

Eq. (5) presents a generalization of London theory including surface effects. Physically, the influence of both Andreev bound states and a gap suppression at the surface on the electrodynamics of the superconductor are completely represented by the function $f$. Due to translational symmetry $f$ only depends on the distance $x$ to the boundary. In Fig. 1 we show our results for $f(x)$. For the $d$-wave orientation $\alpha=0$ neither Andreev bound states nor a suppression of the gap exist at the boundary and $f$ vanishes identically. In this case London electrodynamics is valid everywhere in the superconducting half-space. For the orientation $\alpha=\pi / 4$ however, there are significant changes of the current response close to the boundary, since the transport is dominated by anomalous Meissner currents of the zero-energy Andreev bound states. The net current response $1-f$ may even become negative, and this effect is enhanced for lower temperatures since the spectral weight of Andreev bound states strongly increases [9, 10]. A few coherence lengths into the bulk London electrodynamics is recovered. An important aspect of the resulting Eq. (5) is, that the thermodynamic and electrodynamic selfconsistency conditions are effectively decoupled. As mentioned above, the function $f$ can be obtained using the gap equation (2) only, without any electrodynamics considered. On the other hand, once 
$f$ is provided, the magnetic vector potential $\mathbf{A}$ of the fully selfconsistent problem may simply be found from Eq. (4) and the modified London equation (5), together with Neumann boundary conditions.

In the following, we show how to find the resulting magnetic field and derive the modified surface barrier. For this purpose it is useful to employ the subscript notation $\mathbf{B}=\mathbf{B}_{L}+\mathbf{B}_{R}$, where the subscript $L$ refers to the well-known London solution of the problem, whereas $R$ denotes the unknown small 'response' term generated by the function $f$ in Eq. (5) due to the presence of Andreev bound states. The London solution itself is given by $\mathbf{B}_{L}=\mathbf{B}_{L}^{W}+\mathbf{B}_{L}^{V}$ with $\mathbf{B}_{L}^{W}(\mathbf{r})=B_{0} e^{-x / \lambda} \mathbf{z}$ and $\mathbf{B}_{L}^{V}(\mathbf{r})=\frac{\Phi_{0}}{2 \pi \lambda^{2}}\left[K_{0}\left(\frac{\left|\mathbf{r}-\mathbf{r}_{v}\right|}{\lambda}\right)-K_{0}\left(\frac{\left|\mathbf{r}-\mathbf{r}_{v}^{*}\right|}{\lambda}\right)\right] \mathbf{z}$ where $\Phi_{0}$ is the flux quantum, $K_{0}$ is a modified Bessel function and the star operator shall define a mirror operation with respect to the boundary, i.e. $\mathbf{r}^{*}=(-x, y)$. Via the superscript notation a field is uniquely divided into two parts. The superscript $W$ refers to the 'wall' part, which is translationally invariant along the boundary and is due to the screened external magnetic field. The 'vortex' part denoted by $V$ incorporates the fields due to vortex and its mirror antivortex. The corresponding magnetic vector potential of the London solution is found from $\mathbf{A}_{L}=-\lambda^{2} \nabla \times \mathbf{B}_{L}$. Then, Eqs. (44) and (5) may be combined to

$$
\left(\Delta-\frac{1}{\lambda^{2}}\right) \mathbf{B}_{R}(\mathbf{r})=-\frac{1}{\lambda^{2}} \nabla \times[f(x) \mathbf{A}(\mathbf{r})] .
$$

Inversion of the differential operator leads to an integration over the superconducting area $S$ that can be written in the form

$$
\mathbf{B}_{R}(\mathbf{r})=\frac{1}{\lambda^{2}} \int_{S} d^{2} r^{\prime} f\left(x^{\prime}\right)\left[\nabla^{\prime} G_{S}\left(\mathbf{r}, \mathbf{r}^{\prime}\right)\right] \times \mathbf{A}\left(\mathbf{r}^{\prime}\right),
$$

where the appropriate Green's function $G_{S}$ of the problem has been involved, which is given by $G_{S}\left(\mathbf{r}, \mathbf{r}^{\prime}\right)=$ $-\frac{1}{2 \pi}\left[K_{0}\left(\frac{\left|\mathbf{r}-\mathbf{r}^{\prime}\right|}{\lambda}\right)-K_{0}\left(\frac{\left|\mathbf{r}-\mathbf{r}^{\prime *}\right|}{\lambda}\right)\right]$. In particular, the unknown magnetic field $\mathbf{B}_{R}$ according to Eq. (7) fulfils Eq. (6), is divergence-free and vanishes everywhere at the boundary. The integrand in Eq. (7) is weighted by the function $f$, which is effectively non-zero only up to a finite distance $l_{f} \ll \lambda$ from the boundary, where the standard London theory is not valid (cf. Fig. 11). Furthermore, also the total deviation from standard London theory is small, i.e. $\int d x f \ll \lambda$. Thus, concentrating on the main contribution to $\mathbf{B}_{R}$, the source term $\mathbf{A}$ in the integrand can be replaced by the London solution $\mathbf{A}_{L}$. Moreover it is sufficient to keep the dominating term only: $B_{R}(\mathbf{r})=\frac{1}{\lambda^{2}} \int_{S} d^{2} r^{\prime} f\left(x^{\prime}\right)\left[\partial_{x^{\prime}} G_{S}\left(\mathbf{r}, \mathbf{r}^{\prime}\right)\right] A_{L, y}\left(\mathbf{r}^{\prime}\right)$. This formula allows to calculate, how the magnetic field deviates from the London solution due to the presence of Andreev bound states.

Let us derive the modified BL barrier now. The corresponding free energy density $g$ of our system is given by $g=g_{0}+\frac{1}{8 \pi}\left[\frac{1}{\lambda^{2}}(1-f) \mathbf{A}^{2}+\mathbf{B}^{2}-2 \mathbf{B}_{0} \cdot \mathbf{B}\right]$. Here, $g_{0}$ denotes the value in the absence of magnetic fields and currents. A rather long but straightforward analysis yields the resulting total free energy $G$ (per unit length of the $z$-direction) as a function of the vortex distance $x_{v}$ :

$$
\begin{aligned}
G\left(x_{v}\right) & =\frac{\Phi_{0}}{8 \pi}\left[2 B_{0} e^{-x_{v} / \lambda}-\frac{\Phi_{0}}{2 \pi \lambda^{2}} K_{0}\left(2 x_{v} / \lambda\right)\right] \\
& +\frac{\Phi_{0}}{8 \pi}\left[B_{R}\left(\mathbf{r}_{v}\right)-\frac{B_{0}}{\Phi_{0}} \int_{S} d^{2} r B_{R}^{V}(\mathbf{r})\right] .
\end{aligned}
$$

Here, an arbitrary additive constant has been chosen such that $G\left(x_{v} \rightarrow \infty\right)=0$. Note, that the first two terms are the original BL barrier (cf. [3]). The latter terms are modifications which only appear when $f \neq 0$ and thus $B_{R} \neq 0$, correspondingly. After some algebra, we find

$$
\begin{aligned}
B_{R}^{W}\left(\mathbf{r}_{v}\right) & =\frac{B_{0}}{2 \lambda} e^{-x_{v} / \lambda} \int_{0}^{\infty} d x^{\prime} f\left(x^{\prime}\right)\left(1+e^{-2 x^{\prime} / \lambda}\right) \\
B_{R}^{V}\left(\mathbf{r}_{v}\right) & =-\frac{\Phi_{0}}{4 \pi \lambda^{3}} \int_{0}^{\infty} d x^{\prime} f\left(x^{\prime}\right) \tilde{K}\left(x_{v}, x^{\prime}\right)
\end{aligned}
$$

and $B_{R}^{W}\left(\mathbf{r}_{v}\right)=-\frac{B_{0}}{\Phi_{0}} \int_{S} d^{2} r B_{R}^{V}(\mathbf{r})$ where the abbreviation $\tilde{K}\left(x_{v}, x^{\prime}\right)=K_{1}\left(2 \frac{x_{v}-x^{\prime}}{\lambda}\right)+2 K_{1}\left(\frac{2 x_{v}}{\lambda}\right)+K_{1}\left(2 \frac{x_{v}+x^{\prime}}{\lambda}\right)$ has been used and we restricted ourselves to $x_{v}>l_{f}$, i.e. the vortex is not directly situated in the small surface region with $f \neq 0$. Since the function $f$ is nonzero only in a small surface region, the above expressions can be further simplified, if one replaces $f\left(x^{\prime}\right)$ by a delta function $c_{f} \delta\left(x^{\prime}\right)$ with $c_{f}=\int_{0}^{\infty} d x^{\prime} f\left(x^{\prime}\right)$. The quantity $c_{f}$ has the dimension of a length and remains the only parameter describing the influence of the Andreev bound states. Then, the final result for the normalized free energy of Eq. (8) is

$$
\hat{G}\left(x_{v}\right)=2 \hat{B}_{0}\left(1+\hat{c}_{f}\right) e^{-\hat{x}_{v}}-\left[K_{0}\left(2 \hat{x}_{v}\right)+2 \hat{c}_{f} K_{1}\left(2 \hat{x}_{v}\right)\right] .
$$

Here, the normalized $\hat{G}$ is defined as $G /\left(\Phi_{0}^{2} / 16 \pi^{2} \lambda^{2}\right), \hat{B}_{0}$ denotes the applied magnetic field in units of $\Phi_{0} / 2 \pi \lambda^{2}$, and we abbreviate $\hat{x}_{v}=x_{v} / \lambda$ and $\hat{c}_{f}=c_{f} / \lambda$.

Eq. (9) is our generalization of the surface barrier. For $\hat{c}_{f}=0$ it reduces to the result of Bean and Livingston [3, 4]. In the presence of Andreev bound states, however, there are two modifications due to $\hat{c}_{f} \neq 0$. The first modification is an enhancement of the repulsive part of the potential, effectively increasing the external magnetic field amplitude. Secondly, also the attractive part of the potential coming from the antivortex is strengthened by an additional term. Note, that $\hat{c}_{f} \ll 1$. Even the maximum value of Fig. 1 1 occuring for the orientation $\alpha=\pi / 4$ at the temperature $T=0.1 T_{c}$ is only $c_{f} \approx 3.71 \xi$, i.e. $\hat{c}_{f} \approx 3.71 \kappa^{-1}$. Nevertheless particularly the second term becomes important, because it contains a Bessel function, which diverges for small vortex distances $x_{v}$.

It is important to realize, how drastic some properties may change just because of this rather small looking 


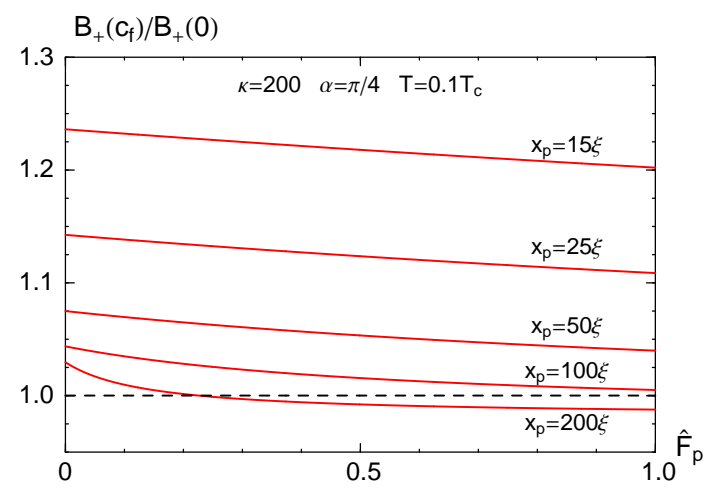

FIG. 2: (Color online) Relative change of the external magnetic field $B_{+}$needed to release a vortex from a pinning center towards the bulk. Results are shown as a function of the normalized pinning force $\hat{F}_{p}$ for different positions $x_{p}$ of the pinning center. Obviously, unpinning the vortex in the presence of surface bound states requires a significantly higher external magnetic field.

modification. To give an example we consider a vortex pinned in a pinning center at the distance $x_{p}$ from the boundary. The maximum pinning force of that center, measured in units of $\Phi_{0}^{2} / 8 \pi^{2} \lambda^{3}$, is characterized by the parameter $\hat{F}_{p}$. If the applied magnetic field then exceeds some specific value $B_{+}$, which can be determined from Eq. (9), the vortex gets unpinned and proceeds towards the bulk. The relative change for this depinning field is given by

$$
\frac{B_{+}\left(c_{f}\right)}{B_{+}(0)}=\frac{1+\frac{K_{1}\left(2 \hat{x}_{p}\right)}{K_{1}\left(2 \hat{x}_{p}\right)+\hat{F}_{p}}\left(2 \hat{c}_{f} \frac{K_{0}\left(2 \hat{x}_{p}\right)}{K_{1}\left(2 \hat{x}_{p}\right)}+\frac{c_{f}}{x_{p}}\right)}{1+\hat{c}_{f}} .
$$

For the parameters $\kappa=200$ and $c_{f}=3.71 \xi$ from above, some results are shown in Fig. 2, Clearly, the external magnetic field needed to unpin a vortex close to a boundary which exhibits Andreev bound states is significantly enhanced in the most relevant cases.

Analogous to the classical BL work [3], we can roughly estimate the field of first free vortex entry $B_{s}$ by choosing $\hat{F}_{p}=0$ and a distance $x_{v} \sim \xi$ from the boundary. Then, we find

$$
\frac{B_{s}\left(c_{f}\right)}{B_{s}(0)} \approx 1+\frac{c_{f}}{\xi}
$$

where we have assumed $\hat{c}_{f} \ll 1$, but $c_{f} / \xi \sim 1$. Here, $B_{s}(0)$ is the $\mathrm{BL}$ value. This result means that the field of first vortex entry is significantly enhanced by the presence of Andreev bound states. Compared to the classical BL value it may increase by a factor 4-5 for low temperatures and $\alpha=\pi / 4$. In Fig. 3 we show the temperature dependence of $c_{f} / \xi$. The corresponding characteristic change of $B_{s}$ as a function of temperature, which is indicated by the inset of Fig. 3. may facilitate experimental observation of this effect.

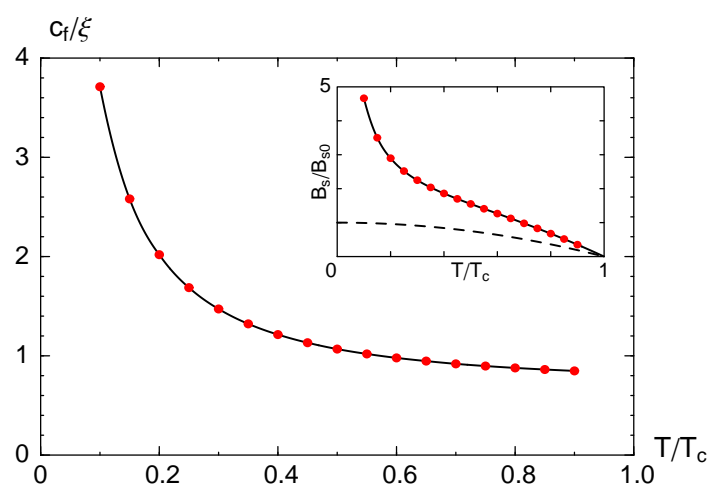

FIG. 3: (Color online) Temperature dependence of the parameter $c_{f} / \xi$ for $\alpha=\pi / 4$. The inset shows the expected temperature dependence of the field of first vortex entry $B_{s}$ according to Eq. (11) (solid line) compared with the BL result (dashed line).

We also expect the modification of the BL barrier to have a strong influence on the hysteresis of the split of the zero bias conductance peak as observed in tunneling experiments [17, 18]. However, a detailed calculation of the hysteresis curve goes beyond the scope of the present work.

[1] T. Kibble, Physics Today 60, No. 9, p. 47 (2007).

[2] L. Kramer and W. Pesch, Z. Physik 269, 59 (1974).

[3] C.P. Bean and J.D. Livingston, Phys. Rev. Lett. 12, 14 (1964).

[4] P.G. de Gennes, Superconductivity of Metals and Alloys (Benjamin, New York, 1966).

[5] C.R. Hu, Phys. Rev. Lett. 72, 1526 (1994).

[6] Y. Tanaka and S. Kashiwaya, Phys. Rev. Lett. 74, 3451 (1995).

[7] L.J. Buchholtz, M. Palumbo, D. Rainer, and J.A. Sauls, J. Low Temp. Phys. 101, 1099 (1995).

[8] S. Kashiwaya and Y. Tanaka, Rep. Prog. Phys. 63, 1641 (2000).

[9] M. Fogelström, D. Rainer, and J.A. Sauls, Phys. Rev. Lett. 79, 281 (1997).

[10] H. Walter et al., Phys. Rev. Lett. 80, 3598 (1998).

[11] S.Graser, C.Iniotakis, T.Dahm, and N.Schopohl, Phys. Rev. Lett. 93, 247001 (2004).

[12] G. Eilenberger, Z. Phys. 214, 195 (1968).

[13] A.I. Larkin and Yu.N. Ovchinnikov, Zh. Éksp. Teor. Fiz. 55, 2262 (1968); Sov. Phys. JETP 28, 1200 (1969).

[14] J.W. Serene and D. Rainer, Phys. Rep. 101, 221 (1983).

[15] N. Schopohl and K. Maki, Phys. Rev. B 52, 490 (1995); N. Schopohl, cond-mat/9804064 (1998).

[16] M. Eschrig, Phys. Rev. B 61, 9061 (2000).

[17] M. Aprili, E. Badica, and L. H. Greene, Phys. Rev. Lett. 83, 4630 (1999).

[18] R. Krupke and G. Deutscher, Phys. Rev. Lett. 83, 4634 (1999). 